\title{
Brane Gravity from Bulk Vector Field
}

\author{
Merab Gogberashvili \\ Andronikashvili Institute of Physics, 6 Tamarashvili Str., Tbilisi 380077, Georgia \\ E-mail: gogber@hotmail.com
}

October 26, 2018

\begin{abstract}
It is shown that Einstein's equations on the brane can be received from the multi-dimensional vector field equations in pseudo-Euclidean space. The idea is based on the observation that the brane geometry can be equivalently described by the intrinsic metric or by the derivatives of its normal. From the other hand the normal to the brane can be constructed with the components of some multidimensional vector fields. For the both cases 4-dimensional effective action for gravity appears to be the same.

PACS numbers: 04.50.+h, 03.50.De, 98.80.Cq
\end{abstract}

Since the over 80 years old paper of Theodor Kaluza [1] it has been believed that Einstein's equations are more fundamental then matter fields equations. In standard Kaluza-Klein approach matter fields are considered to be a part of multi-dimensional metric tensor. However, the difficulties of General Relativity are well known and it is still not clear whether gravity is a quantum field or some classical effective interaction.

Recently, it was shown that Plank's scale $M_{P l}$ can be constructed with the fundamental scale $M$ and the brane width $\epsilon$ [2]. On the brane, possibly, not only $M_{P l}$, but Einstein equations can be effective as well. In multi dimensions the equations describing gravity can be quite different from Einstein's equations.

Note that the first published suggestion about the unification of gravity and matter in multi dimensions (which was given by Gunnar Nordstrom [3] anticipating even General Relativity) was based on vector fields approach. Nordstrom started from 5-dimensional Maxwell's equations and imposed also the 'cilinder condition' (the fields should not depend on the fifth coordinate). He identified an extra component of vector-potential with the gravity potential required in his scalar gravity theory. In some recent papers these extra scalar components of multi-dimensional vector-potentials are used to explain Higgs mechanism on the brane [州.

In this paper we also use a multi-dimensional vector field to describe gravity on the brane, but do not follow the simple approach of Nordstrom. When in pseudo-Euclidean space a brane is embedded, multidimensional vector fields together with the brane geometry can imitate Einstein gravity on the brane. Here we don't specify the nature of the brane, for example, it can be the kink solution of nonlinear equation of some multi-dimensional scalar field. We just show that solutions of 4-dimensional Einstein's equations could be constructed with the solutions of multidimensional Maxwell's equations. In this picture gravity exhibits tensor character only on the brane and graviton appears to be the combination of two 1-spin massless particles. We hope that in such approach geometrical unification of different interactions will be easier since Dirac equation also can be derived from the constrained Yang-Mills Lagrangian 5. Similar ideas inducing gravity (in the linear approximation) on some plane in multi dimensions was considered in [6] using analogies with elasticity theory.

We want to start with reminding the reader that any $n$-dimensional Riemannian space can be embedded into $N$-dimensional pseudo-Euclidean space with $n \leq N \leq n(n+1) / 2$ [7]. Thus, no more than ten dimensions are required to embed any 4-dimensional solution of Einstein's equations with arbitrary energy-momentum tensor. Embedding the space-time with the coordinates $x^{\alpha}$ and metric $g_{\alpha \beta}$ into pseudo-Euclidean space with Cartesian coordinates $\phi^{A}$ and Minkowskian metric $\eta_{A B}$ is given by

$$
d s^{2}=g_{\alpha \beta} d x^{\alpha} d x^{\beta}=\eta_{A B} h_{\alpha}^{A} h_{\beta}^{B} d x^{\alpha} d x^{\beta}=\eta_{A B} d \phi^{A} d \phi^{B} .
$$


Capital Latin letters $A, B, \ldots$ labels coordinates of embedded space, while Greek indices $\alpha, \beta, \ldots$ enumerate coordinates in four dimensions. Existence of the embedding (11) demonstrates that the multidimensional 'tetrad' fields $h_{\alpha}^{A}$ can be expressed as a derivatives of some vector

$$
h_{\alpha}^{A}=\partial_{\alpha} \phi^{A} .
$$

In four dimensions when tetrad index run over only four values such relation is impossible in general and according to (1) it could be always written in multi dimensions.

Let's suppose that in multi-dimensional flat space-time there exists $(1+3)$-brane with arbitrary geometry. In order to simplify demonstration of the idea, let us first consider the case of only one extra space-like dimension. Generalization for arbitrary dimensions and signature is obvious.

Let's say that the equation of the branes surface in the Cartesian 5-dimensional coordinates $X^{A}$ has the form:

$$
W\left(X^{A}\right)=0 .
$$

Introducing the function

$$
\xi\left(X^{A}\right)=\frac{W\left(X^{A}\right)}{\sqrt{\left|\partial_{B} W \partial^{B} W\right|}},
$$

the metric of pseudo-Euclidean bulk $(1+4)$-space can be transformed to the Gaussian normal coordinates

$$
d s^{2}=-d \xi^{2}+g_{\alpha \beta}\left(\xi, x^{\nu}\right) d x^{\alpha} d x^{\beta} .
$$

Since $\xi=0$ is the equation of the hyper-surface, the induced metric $g_{\alpha \beta}\left(0, x^{\nu}\right)$, which determines the geometry on the brane, is the same 4-dimensional metric as used in (1) for the embedding.

Introducing unit normal vector to the brane

$$
n^{A}=\left.\partial^{A} \xi\right|_{\xi=0},
$$

one can decompose tensors of bulk space-time in a standard way (see e. g. [8]).

In the Gaussian system of coordinates (5) the Christoffel symbols on the brane are:

$$
\Gamma_{\nu \lambda}^{\alpha}=h^{A \alpha} \partial_{\lambda} h_{A \nu} .
$$

Raising and lowering of Greek indices is made with the induced metric tensor $g_{\alpha \beta}$ and Latin indices with 5-dimensional Minkowskian metric tensor $\eta_{A B}$. The Christoffel symbols containing two or three indices $\xi$ are equal to zero. The connections containing just one index $\xi$ are forming outer curvature tensor, which, after using (2), can be written as:

$$
K_{\alpha \beta}=n_{A} D_{\beta} h_{\alpha}^{A}=-\Gamma_{\alpha \beta}^{\xi}=\partial_{\alpha} \partial_{\beta} \phi^{\xi},
$$

where $D_{\beta}$ denotes covariant derivatives in Gaussian coordinates (5) and $\phi^{\xi}$ is the transversal component of the embedding function.

Since bulk 5-dimensional space-time is pseudo-Euclidean, its scalar curvature is zero:

$$
{ }^{5} R=R+K^{2}-K_{\alpha \beta} K^{\alpha \beta}=0 .
$$

From this relation the 4-dimensional scalar curvature $R$ can be expressed with the quadratic combinations of the extrinsic curvature $K_{\alpha \beta}$. Thus, using (8) Hilbert's 4-dimensional gravitational action (after removing of boundary terms) can be written in the form:

$$
S_{g}=-M_{P l}^{2} \int R \sqrt{-g} d^{4} x=M_{P l}^{2} \int\left(\square \phi^{\xi} \square \phi^{\xi}-\partial_{\alpha} \partial_{\beta} \phi^{\xi} \partial^{\alpha} \partial^{\beta} \phi^{\xi}\right) \sqrt{-g} d^{4} x,
$$

where $\square=\partial_{\alpha} \partial^{\alpha}$ is the 4-dimensional wave operator and $g$ is the determinant in Gaussian coordinates. It is clear now that embedding theory allows us to rewrite 4-dimensional gravitational action in terms of derivatives of the normal components of some multi-dimensional vector. 
Now let us consider the bulk massless vector field $A^{B}$ that obeys 5-dimensional Maxwell's equations

$$
\partial_{A} F^{A B}=0
$$

where $F_{A B}=\partial_{A} A_{B}-\partial_{B} A_{A}$ is the ordinary field strength. We avoid connection of the functions $A^{B}$ with the bulk coordinates $X^{A}$, not to restrict ourselves with pure geometrical interpretation.

The action for Maxwell's field can be written in the general form:

$$
S_{A}=-\frac{1}{4} \int F_{A B} F^{A B} d^{5} X=-\frac{1}{2} \int\left[\partial_{A} A_{B}\left(\partial^{A} A^{B}+\partial^{B} A^{A}\right)-2 \partial_{A} A^{A} \partial_{B} A^{B}\right] d^{5} X .
$$

We shall demonstrate below that on the brane this action can be reduced to the 4-dimensional gravity action (10).

Note that so called vacuum gauge fields - the solutions of the equations

$$
F_{A B}=0,
$$

are always present in the space-time. These fields are solutions of Maxwell's equations (11) as well. If there are no topological defects in space-time, the solutions of (13) are pure gauges. The example of non-trivial solution of (13) in space-time with the linear defect is Aharonov-Bohm field ( see e. g. [9]). For the brane the normal to its surface components of vacuum gauge fields also are non-trivial and we shall show that they can resemble gravity on the brane.

In the Gaussian coordinates (5) the ansatz which satisfies (13) and has the symmetries of the brane, with the accuracy of constants, can be written in the form:

$$
A^{\alpha}=G(\xi) \partial^{\alpha} \phi\left(x^{\beta}\right), \quad A^{\xi}=\phi\left(x^{\beta}\right) \partial^{\xi} G(\xi),
$$

where $G(\xi)$ and $\phi\left(x^{\beta}\right)$ are some functions depending on the fifth coordinate $\xi$, and four-coordinates $x^{\beta}$ respectively. We assume that $G(\xi)$ is an even function of $\xi$ and the integrals from $G(\xi)$ and from its derivative are convergent. Simple example of such a function is $\exp \left(\xi^{2}\right)$. So, we choose the ansatz:

$$
A^{\alpha}=\frac{c}{\epsilon^{3 / 2}} \partial^{\alpha} \phi^{\xi}\left(x^{\beta}\right) \exp \left(-\frac{\xi^{2}}{\epsilon^{2}}\right), \quad A^{\xi}=-\frac{2 c}{\epsilon^{7 / 2}} \xi \phi^{\xi}\left(x^{\beta}\right) \exp \left(-\frac{\xi^{2}}{\epsilon^{2}}\right),
$$

where $\epsilon$ is the brane width and $c$ is some dimensionless constant. We assume that the width of the brane $\epsilon$ is constant all along the surface.

Inserting the ansatz (15) into action integral (12), and integrating by the normal coordinate $\xi$ we receive the induced action on the brane:

$$
S_{A}=\sqrt{\frac{\pi}{2}} \frac{c^{2}}{\epsilon^{2}} \int\left(\square \phi^{\xi} \square \phi^{\xi}-\partial_{\alpha} \partial_{\beta} \phi^{\xi} \partial^{\alpha} \partial^{\beta} \phi^{\xi}\right) \sqrt{-g} d^{4} x,
$$

where summing is made with the intrinsic metric $g_{\alpha \beta}\left(x^{\nu}\right)$. If we put

$$
M_{P l}^{2}=\sqrt{\frac{\pi}{2}} \frac{c^{2}}{\epsilon^{2}}
$$

the effective action of 5-dimensional vector field (16) becomes equivalent to Hilbert's action for 4dimensional gravity (10). So, 4-dimensional Einstein's equations on the brane can be received from Maxwell's multi-dimensional equations in flat space-time.

The same result can be obtained in the case $N>5$. Now $\xi$ and $\phi^{\xi}$ in (6), (8) and (15) must be replaced by $\xi^{i}$ and $\phi^{i}$, and action integrals (10) and (16) transform to the sum:

$$
S_{\Sigma}=M_{P l}^{2} \eta_{i j} \int\left(\square \phi^{i} \square \phi^{j}-\partial_{\alpha} \partial_{\beta} \phi^{i} \partial^{\alpha} \partial^{\beta} \phi^{j}\right) \sqrt{-g} d^{4} x,
$$

where $\eta_{i j}$ is the Minkowskian metric of the normal space to the brane. Small Latin indices $i, j, \ldots$ enumerates extra $(N-4)$ coordinates. If we assume that all the brane widths are equal to $\epsilon$ for the scale in (18) we have:

$$
M_{P l}^{2}=\frac{c^{2}}{\epsilon^{2}}\left(\frac{\pi}{2}\right)^{(N-4) / 2}
$$


Now we want to consider the particular example 6-dimensional pseudo-Euclidean space with the signature $(2+4)$ to demonstrate 4-dimensional unification of gravity and Electro-Magnetism based on Maxwell's equations. In (18) there exists only derivatives of $\phi^{i}$ (which we connected with gravity) and it is clear that $F_{\alpha \beta}$ is zero. Now we want to insert in (15) also the non-integrable part $a^{\alpha}\left(x^{\beta}\right)$ and identify it with the 4-dimensional vector-potentials.

The $(2+4)$-space is interesting object for investigations. As it was found a long time ago, in this space Schwarzschild's metric admits embedding [10], and that non-linear 15-parameter conformal group can be written as a linear Lorentz-type transformations 11]. Another fact is that 5-dimensional metric with exponential warp factor (needed to cancel cosmological constant [12]) can be embedded in the Euclidean $(2+4)$-space [13]. For compact Kaluza-Klein models the (2+4)-space was studied in [14 and in the context of brane models with non-factorizable geometry in [15].

Let us insert $a^{\alpha}\left(x^{\beta}\right)$ in (15) and rewrite it in the form:

$$
\begin{array}{r}
A^{\alpha}=\frac{c}{\epsilon^{2}} \partial^{\alpha} \phi^{\tau}\left(x^{\beta}\right) \exp \left(-\frac{\tau^{2}}{\epsilon^{2}}\right)+\frac{c}{\epsilon^{2}} \partial^{\alpha} \phi^{\kappa}\left(x^{\beta}\right) \exp \left(-\frac{\kappa^{2}}{\epsilon^{2}}\right)+a^{\alpha} \exp \left(-\frac{\tau^{2}+\kappa^{2}}{\epsilon^{2}}\right), \\
A^{\tau}=-\frac{2 c}{\epsilon^{4}} \tau \phi^{\tau}\left(x^{\beta}\right) \exp \left(-\frac{\tau^{2}}{\epsilon^{2}}\right), \\
A^{\kappa}=-\frac{2 c}{\epsilon^{4}} \kappa \phi^{\kappa}\left(x^{\beta}\right) \exp \left(-\frac{\kappa^{2}}{\epsilon^{2}}\right),
\end{array}
$$

where $\kappa$ and $\tau$ are the normal to the brane space-like and time-like coordinates respectively.

Using the ansatz (20) Maxwell's action in (2+4)-space takes the form:

$$
\begin{array}{r}
S_{A}=-\frac{1}{4} \int F_{A B} F^{A B} d^{6} X= \\
=-\frac{\pi c^{2}}{2 \epsilon^{2}} \int R \sqrt{-g} d^{4} x-\frac{1}{4 \epsilon^{2}} \int \exp \left[-\frac{2\left(\tau^{2}+\kappa^{2}\right)}{\epsilon^{2}}\right]\left[f_{\alpha \beta} f^{\alpha \beta}+\frac{4}{\epsilon^{4}}\left(\tau^{2}-\kappa^{2}\right) a_{\alpha} a^{\alpha}\right] \sqrt{-g} d^{4} x d \tau d \kappa= \\
=-\int\left(M_{P l}^{2} R+\frac{1}{4} f_{\alpha \beta} f^{\alpha \beta}\right) \sqrt{-g} d^{4} x,
\end{array}
$$

where $f_{\alpha \beta}=\partial_{\alpha} a_{\beta}-\partial_{\beta} a_{\alpha}$. So, 6-dimensional Maxwell's action on the brane is reduced to the 4-dimensional Einstein-Maxwell action. As in 15] the brane is placed at $\tau^{2}=\kappa^{2}$, that means that it moves with the speed of light in transversal $(1+1)$-space.

In Gaussian coordinates (5) Maxwell's 6-dimensional equations, after inserting there the ansatz (20), reduces to the system:

$$
\begin{aligned}
\frac{1}{\sqrt{-g}} \partial_{\alpha}\left(\sqrt{-g} f^{\alpha \beta}\right)-\frac{4}{\epsilon^{4}}\left(\tau^{2}-\kappa^{2}\right) a^{\beta} & =0, \\
\partial_{\alpha} a^{\alpha} & =0 .
\end{aligned}
$$

One can see that the situation is similar to superconductors. On the brane $\left(\tau^{2}=\kappa^{2}\right)$ photon is massless and obtains the large mass $\sim 1 / \epsilon$ in the direction normal to the brane.

We want to note that even if we don't want to connect $\phi^{i}$ with gravity, the ansatz (20) can be used for trapping photons on the brane in the (2+4)-space (for localization of massless vector fields on a brane, see [15, 16]).

Hence, in this paper the equivalence of the descriptions of 4-dimensional gravity both with the intrinsic metric, and with the multi-dimensional vector field was demonstrated. We have not considered here models for the field that forms the brane, as well as the question how the brane geometry is changed because of couplings with 4-dimensional matter was not raised.

Acknowledgements: Author would like to acknowledge the hospitality extended during his visits at the Abdus Salam International Centre for Theoretical Physics where this work was done.

\section{References}

[1] T. Kaluza, Sitz. Preuss. Akad. der Wiss., 96, 69 (1921). 
[2] N. Arkani-Hamed, S. Dimopoulos and G. Dvali, Phys. Lett. B429, 263 (1998);

I. Antoniadis, N. Arkani-Hamed, S. Dimopoulos and G. Dvali, Phys. Lett. B436, 257 (1998).

[3] G. Nordstrom, Phys. Zeitsch., 15, 504 (1914).

[4] G. Dvali, S. Randjbar-Daemi and R. Tabbash, Phys. Rev., D 65, 064021 (2002);

M. Shaposhnikov and P. Tinyakov, Phys. Lett., B 515, 442 (2001).

[5] Y. Takahashi, J. Math. Phys., 24, 1783 (1983);

V. A. Zhelnorovich, Proc. Acad. Sci. USSR, 311, 590 (1990);

F. Reifler and R. Morris, J. Math. Phys., 36, 1741 (1995).

[6] S. S. Kokarev, Nuovo Cim., 113, 1339 (1998); 114, 903 (1999); 116, 915 (2001).

[7] L. P. Eisenhart, Riemannian Geometry (New Jersey: Princeton University Press, 1949);

A. Friedman, J. Math. Mech., 10, 625 (1961); Rev. Mod. Phys., 37, 201 (1965).

[8] C. W. Misner, K. S. Thorne and J. A. Wheeler, Gravitation (San Francisco: Freeman, 1973).

[9] M. Peskin and A. Tonomura, The Aharonov-Bohm Effect (Berlin: Springer-Verlag, 1989).

[10] J. Rosen, Rev. Mod. Phys., 37, 204 (1965).

[11] P. Dirac, Ann. Math., 37, 429 (1936); Proc. Roy. Soc., A155, 447 (1936).

[12] M. Gogberashvili, hep-ph/9812296; Mod. Phys. Lett., A14, 2024 (1999); Europhys. Lett., 49, 396 (2000); hep-ph/9908347;

L. Randall and R. Sundrum, Phys. Rev.Lett., 83, 3370 (1999); 83, 4690 (1999).

[13] M. Gogberashvili, J. Math. Phys., 43, 4886 (2002).

[14] M. Pavsic, Nuovo Cimento, B41, 397 (1977);

R. L. Ingraham, Nouvo Cimento, B46, 1 (1978); B46, 16 (1978); B46, 217 (1978); B46, 261 (1978);

B47, 157 (1978); B50, 233 (1979); B68, 203 (1982); B68, 218 (1982).

[15] M. Gogberashvili, Phys. Lett., B484, 124 (2000);

M. Gogberashvili and P. Midodashvili, Phys. Lett., B515, 447 (2001); hep-th/0111132.

[16] G. Dvali and M. Shifman, Phys. Lett., B 396, 64 (1997);

B. Bajc and G. Gabadadze, Phys. Lett, B 474, 282 (2000);

A. Pomarol, Phys. Lett., B 486, 153 (2000);

I. Oda, Phys. Rev., D 62, 126009 (2000). 